\title{
Arbor
}

\section{La rehabilitación en el deporte}

\author{
Jesús Olmo Navas
}

Arbor CLXV, 650 (Febrero 2000), 227-248 pp.

Las lesiones deportivas presentan una creciente prevalencia, asociada con el aumento de la práctica deportiva. Tanto a niveles de competición como recreacionales, se constata una demanda del deportista para volver a la actividad de la forma más rápida y funcional posible. Se examinan en este trabajo las características de la lesión de aparato locomotor en el deporte, y las peculiaridades del proceso de rehabilitación tras el tratamiento inicial, analizándose los actuales procedimientos e infraestructura empleados para su desarrollo, desde una perspectiva multidisciplinar.

\section{Introducción: la lesión deportiva, su prevención y su rehabilitación}

El deporte, en sus diferentes manifestaciones, constituye un elemento de primer orden en la estructura de la mayoría de las sociedades humanas. Los individuos de nuestra especie han competido atléticamente entre sí, y también contra ellos mismos a través de la historia conocida, y como consecuencia, han experimentado lesiones durante la preparación o la competición en sí misma.

Una lesión deportiva puede ser definida ampliamente como cualquier lesión que está relacionada con la actividad física y. que resulta en el mantenimiento del individuo fuera del entrenamiento, actividad o competición el día siguiente del episodio; también puede definirse como cualquier lesión relacionada con el deporte que requiere atención médica. La incidencia de este tipo de patología continúa subiendo entre la población deportista, y también entre la población general, debido al 


\section{Jesús Olmo Navas}

incremento de la práctica de deporte-salud como medida de medicina preventiva para muchas enfermedades.

La lesión deportiva se da mayoritariamente en el sistema musculoesquelético, de manera que entre los profesionales enfocados a esta área de la anatomía humana podemos encontrar a la mayoría de los especialistas. La incidencia principal se agrupa esencialmente en las articulaciones de los miembros inferiores, con la rodilla como la región más frecuentemente afectada. En deportes que demandan la utilización del miembro superior, el hombro es el principal foco de lesiones, debido a la movilidad extensiva y escasa estabilidad inherentes a su estructura.

El estudio, diagnóstico y manejo de estas lesiones ha evolucionado dentro del campo multidisciplinar de la medicina deportiva, interesando a médicos, terapeutas, científicos del deporte, entrenadores y a toda una cohorte de profesionales de la salud, educadores e investigadores que han orientado sus respectivas carreras hacia la prevención, tratamiento y rehabilitación de las lesiones deportivas, con el objetivo siempre de devolver al individuo al más alto nivel de actividad posible.

La prevención es un factor clave para disminuir las posibilidades de lesión. La adecuada progresión, intensidad, frecuencia y carga consiguen motivar un decremento de estas posibilidades, en especial de las lesiones por sobrecarga. Aunque la prevención siempre ha sido objeto de discusión, la realidad es que poco se ha avanzado en este campo. La actitud general hacia su importancia real está gradualmente cambiando hacia un reconocimiento de su papel esencial en el cuidado continuo del deportista, de modo que cada vez hay más número de éstos que incluyen en su planificación de entrenamiento programas específicos y medidas generales de prevención de lesiones, con el consiguiente impacto en el rendimiento general temporada tras temporada.

El objetivo del tratamiento y la rehabilitación de la lesión deportiva es la restauración de la función atlética, en el mayor grado posible, en el tiempo más corto posible. La práctica basada en los conocimientos científicos, focalizada en el manejo del curso temporal de la reacción inflamatoria inicial y los procesos de reparación subsecuentes, reconociendo las características de cicatrización de los tejidos neuromusculares, articulares y óseos, y fundamentada en la apreciación de la mecánica articular, la fisiología del ejercicio, y la psicología del deportista con respecto a su lesión, proporciona sin duda la resolución de muchos de éstos procesos patológicos ${ }^{1}$.

La rehabilitación deportiva viene marcada por el tiempo. El periodo temporal que transcurre mientras un deportista está lesionado afecta negativamente a su condición física y rendimiento posterior. Para cual- 
quier practicante de deporte, tenga el nivel que tenga, las implicaciones personales derivadas del tiempo de inactividad son importantes en la mayoría de los casos. Para un deportista de alto nivel, estas implicaciones pueden ser dramáticas, y trascienden del ámbito de lo personal para pasar al de lo social y económico.

En este aspecto, se observa un progresivo interés en la inclusión de unidades altamente tecnificadas de rehabilitación y prevención de lesiones deportivas dentro del complejo de estructuras que deben acompañar necesariamente al deportista de alto rendimiento. Es evidente que no tiene sentido dedicar unos amplios recursos para optimizar el entrenamiento de un atleta de élite sin pensar en la alta incidencia de lesiones que este tipo de atletas presenta. Una unidad de rehabilitación deportiva de calidad inferior al resto de unidades que configuran la estructura de alto rendimiento puede afectar seriamente a los resultados deportivos del deportista de alto nivel, y representa una carencia tanto o más grave que la ausencia de una buena instalación de entrenamiento o de un buen entrenador. Los países avanzados en este aspecto han reconocido desde hace tiempo la importancia de estos planteamientos, con resultados valorables. Otros países que pugnan por elevar su nivel deportivo internacional están comenzando a integrar este tipo de unidades dentro de su planificación para el alto rendimiento deportivo.

Sin llegar a estos niveles de tecnificación, y asociado al auge de la actividad deportiva en la población general, se observa un cada vez mayor interés en la recuperación adecuada de las lesiones que esta práctica conlleva. No cabe duda que, conforme el nivel de bienestar de la sociedad aumenta, la demanda de una mayor calidad en la rehabilitación de las lesiones se incrementa, siendo éste un hecho que cualquier estructura sanitaria no puede ignorar. La rehabilitación ha pasado de ser una especie de «lujo» a ser solicitada como parte integral del tratamiento de una patología susceptible de su aportación. En el campo del deporte, se constata una exigencia creciente del practicante, ya sea recreacional o competitivo de cualquier nivel, por volver a la práctica de su deporte favorito de la manera más rápida posible. Esta exigencia lleva muchas veces al deportista a desdeñar propuestas científicas de tratamiento basadas en la evidencia, y fundamentadas sobre el respeto de los tiempos fisiológicos de reparación, a favor de ofertas de regímenes de tratamiento no convencionales, y supuestamente más breves, ofrecidos por toda una cohorte de técnicos con conocimientos de la patofisiología de la lesión de aparato locomotor. El estado embrionario de la investigación básica en muchos de los procesos patológicos 
deportivos, asociado a una secular escasa consideración de la importancia de éstos, ha llevado a la proliferación de estas propuestas terapéuticas, de base en muchos casos simplemente empírica, y cuyo valor en muchos casos está por demostrar.

\section{El concepto integral de la rehabilitación en el deporte}

Distintivamente con respecto a la rehabilitación convencional, la rehabilitación deportiva requiere no sólo la completa restauración del rendimiento funcional de la articulación o extremidad afecta, sino que también comprende el mantenimiento de las capacidades atléticas del deportista, mediante su trabajo según un plan de entrenamiento modificado de acuerdo a las características de la lesión.

De este modo, el modelo de plan de tratamiento para una lesión deportiva se asemeja mucho a una planificación de entrenamiento, con los añadidos de la terapéutica necesaria sobre el foco patológico. Este plan se construye sobre el conocimiento de los límites en la regeneración del tejido, y a través de la recuperación de la fuerza, potencia y resistencia musculares, mientras que la flexibilidad estructural y la capacidad cardiovascular se mantienen o mejoran. De este modo, el proceso de rehabilitación se puede estructurar según los principios de la planificación moderna del entrenamiento, dado que se van a trabajar muchas de las cualidades físicas del deportista, con las modificaciones dictadas por su lesión. Esta planificación se puede materializar en el diseño de un macrociclo de rehabilitación, que es caracterizado por el objetivo concreto de la recuperación de la lesión, y con objetivos secundarios de mantenimiento o mejora de otras cualidades físicas. No podemos olvidar que el tiempo de baja es un excelente momento para mejorar áreas de rendimiento del deportista cuyo trabajo se ha podido limitar anteriormente por cuestiones de priorización y gestión de tiempo. De este modo, durante el periodo de lesión se pueden trabajar aspectos como la flexibilidad o la musculación de algunos segmentos corporales, de modo que se consigan mejoras a la hora de la vuelta al entrenamiento. El macrociclo de rehabilitación puede estructurarse en mesociclos de acumulación, transformación y realización, y en microciclos, de acuerdo a los principios de la planificación deportiva contemporánea.

Los objetivos funcionales finales del proceso de rehabilitación suelen ser mucho más ambiciosos en el deportista que en el individuo sedentario, ya que las demandas de aquel sobre su sistema musculoes quelético son mucho mayores. El establecimiento de los objetivos 
rehabilitadores debe pues basarse en un conocimiento profundo de estas demandas, las cuales varían según la especialidad deportiva y que comprenden no sólo un alto grado de desarrollo de las cualidades físicas básicas, sino además unas elevadas prestaciones de control neuromuscular y de funcionalidad deportiva. A este respecto, destaca la importancia del trabajo sobre la propiocepción y sobre la readquisición de las destrezas propias del deporte. Progresando de general a específico, de simple a complejo, de fácil a difícil con incrementos en la intensidad y repetición, el deportista es reintroducido en su actividad deportiva habitual. De este modo, la progresión del deportista a través de su lesión se estructura según unos objetivos parciales complejos, para cuya obtención cada etapa se basa en los progresos obtenidos en la anterior.

Puesto que tanto la lesión del deportista, como el propio deportista en sí, representan problemas complejos, la rehabilitación deportiva necesita integrar numerosas herramientas diagnósticas, terapéuticas y de entrenamiento. Una aproximación multidisciplinar al problema resulta sin duda en la mejor disposición de los esfuerzos ante la patología deportiva, ya que la diversidad de factores que hay que monitorizar y mejorar aconseja la intervención de diferentes expertos. En el campo diagnóstico, se hace necesaria la integración de exploraciones clínicas e instrumentales, con aquellas llamadas funcionales y con tests de rendimiento deportivo seleccionados. La lesión deportiva provoca un proceso de desentrenamiento que es necesario valorar en todas sus áreas. Para ello, es muy interesante la aportación de pruebas físicas estandarizadas propias del medio deportivo. Las evaluaciones debieran ser continuadas, como parte de un proceso de retroalimentación constante, común con el sistema cerrado característico del entrenamiento deportivo moderno. En el campo terapéutico, el gran arsenal de medios disponibles requiere la gestión cuidadosa de elementos tales como la medicación, los medios físicos, la fisioterapia, las ortesis y equipamiento, la nutrición, la corrección biomecánica, la intervención psicológica y la planificación de entrenamiento integrado, entre otros. Cada uno de estos ítems es dominado por diversos tipos de especialistas, cuyo trabajo coordinado es la mejor garantía de eficacia. Enfoques individualistas de la lesión deportiva dan lugar indefectiblemente a carencias importantes en la recuperación del deportista.

\section{Fisiología de la condición física y de la rehabilitación}

Dado que la rehabilitación deportiva emplea un programa de entrenamiento modificado para restablecer la función perdida y mantener 
la condición general, el proceso debiera estar presidido en todo momento por los principios generales del entrenamiento.

El rendimiento de un deportista está influenciado por un gran número de factores, cuya importancia es necesario identificar, priorizar, evaluar y modificar si se quiere sacar el máximo partido a las condiciones individuales. Muchos de los condicionantes que afectan negativamente al rendimiento pueden salir a la luz en el momento de una lesión, y ésta puede ser la consecuencia de aquellos. En numerosas ocasiones, es a partir de una lesión cuando los necesarios cambios en el entrenamiento del sujeto son realizados. De este modo, el proceso de rehabilitación surte un efecto corrector, que trasciende del tiempo de lesión para formar parte de la estrategia de entrenamiento del deportista.

Durante el tiempo en el que el deportista está lesionado, es importante la conservación del mayor número de componentes de la condición física posible. El abandono total de la actividad y la sustitución de ésta por media hora al día de fisioterapia es una práctica bastante frecuente, que condiciona sin duda un mayor tiempo de baja, un rendimiento disminuido al término del proceso y un mayor riesgo de lesión recurrente. No se debería de perder de vista que la condición física es un conjunto complejo de factores que podríamos agrupar convencionalmente en:

1. Sistemas de energía muscular, representados en las vías aeróbica, anaeróbica láctica y anaeróbica aláctica, cuyo desarrollo expresa una potencia y capacidad determinadas para cada una de ellas.

2. Sistema cardiorrespiratorio, en el que se podrían englobar las características de la función respiratoria, cardiaca, circulatoria y hemática.

3. Sistema neuromuscular, el que se incluirían las cualidades musculares de fuerza, potencia y resistencia, junto con factores neurales complejos como el reclutamiento, frecuencia de contracción, la sincronicidad, la secuenciación de patrones de movimiento, el equilibrio o el control de la estabilidad de una articulación. rales.

4. Flexibilidad, con sus componentes articulares, tisulares y neu-

5. Composición corporal, con sus componentes de masa grasa, masa muscular y masa ósea, de importancia relativa al tipo de deporte.

Es fácil comprender como, si la reparación de una lesión dura, por ejemplo, dos meses, estos factores quedarán dramáticamente mermados en caso de que no se les someta a un programa serio de trabajo. Si esto no es así, al alta del deportista nos podemos encontrar con, 
por ejemplo, varios kilos de más y una condición física pobre que requiera un tiempo adicional para volver a ser la que era.

De este modo, se hace absolutamente necesario el entrenamiento continuado de estos factores, de una manera integrada con el programa de rehabilitación. De hecho, algunos deportistas de elite pueden trabajar más horas durante su rehabilitación que durante el entrenamiento normal, cuando no tienen problemas. En condiciones ideales de alto rendimiento, el programa de rehabilitación preside la vida del deportista durante el tiempo en que éste está lesionado. En deportistas de menor nivel, el programa debe adaptarse a los recursos y tiempo de cada individuo, pero no debe obviarse en absoluto, dado que no solamente tiene incidencia sobre el rendimiento posterior, sino también sobre la propia predisposición a lesionarse de nuevo. La práctica habitual de partir de cero tras una lesión tiene un alto coste en lesiones recidivantes, y esto se observa, desgraciadamente, de forma frecuente en la práctica clínica.

Parte importante del programa de rehabilitación la constituye el entrenamiento de la condición muscular, expresada en sus características de fuerza, potencia y resistencia. La mayoría de las lesiones deportivas motivan una afectación de alguna o de todas estas cualidades, cuyo restablecimiento es materia de trabajo común para el equipo de rehabilitación, el cual debiera trabajar en conexión con el entrenador para desarrollar programas, tanto generales como específicos a las demandas del deporte. Para ello, se hace necesario el conocimiento de las adaptaciones que se dan en respuesta al entrenamiento de fuerza, así como de los tipos y sistemas de entrenamiento reconocidos, de la metodología para la documentación de este tipo de entrenamiento, y del diseño de programas individualizados para lesiones específicas.

Por otra parte, para prescribir un programa de entrenamiento, es necesario basarse en una serie de principios. En primer lugar, éste debiera ser individualizado, fundamentándose en la edad biológica, condiciones genéticas, estado de condición física, estado de salud, y estado de fatiga, entre otros factores. Igualmente, es aconsejable optimizar el desarrollo de los componentes de la condición física de acuerdo al tipo de deporte practicado, puesto que no en todos se requieren unos niveles máximos de cada uno de ellos. Además, y según la fase de programación, es demandable un nivel de especificidad concreto, con una adaptación de las características del entrenamiento a los patrones funcionales del deporte en cuestión. No se puede pasar por alto que las respuestas corporales a las cargas de ejercicio son en gran parte específicas a las estructuras y funciones estimuladas. En 
cuanto a la aplicación de cargas, es importante respetar los principios de estímulo y supercompensación mediante el adecuado descanso/descarga, para lo que se hace necesario conocer los tiempos de recuperación de las cualidades musculares para cada tipo de entrenamiento. Una mala planificación en este aspecto es especialmente grave cuando se trabaja en la recuperación de una lesión, puesto que ésta es especialmente sensible a la sobrecarga. La progresión en el ejercicio, manteniendo el efecto de estímulo, también debe ser cuidada al máximo, ya que una estructura lesionada presenta una menor tolerancia a los incrementos bruscos. La periodización del entrenamiento, su monitorización y su evaluación son aspectos igualmente a tener en cuenta. En general, se puede decir que el programa de rehabilitación deportiva se apoya en los pilares básicos del entrenamiento deportivo. Es fácilmente apreciable como el concurso de un especialista formado en este aspecto es de gran valor dentro del contexto integral de la rehabilitación en el deporte.

De igual modo, el trabajo en equipo con el entrenador o educador habitual del deportista es muy deseable. Son desgraciadamente frecuentes los casos en que un deportista pierde los beneficios de su tratamiento obtenidos en la clínica, debido a sesiones de entrenamiento demasiado intensas o no adecuadas para el momento evolutivo de su lesión, en base a una mala coordinación entre el equipo de rehabilitación y el entrenador. Para evitar estas circunstancias, se debería asegurar que el programa de actuaciones es aceptado y consensuado con el entrenador, tanto como con el propio deportista, así como definidas sus tareas en estas actuaciones. No se puede olvidar que es el entrenador quien tiene la responsabilidad principal sobre la actividad deportiva del paciente, $\mathrm{y}$ es quien mejor puede motivar $\mathrm{y}$ dirigir a éste para el cumplimiento del plan de entrenamiento modificado que supone el programa de rehabilitación. Su papel es, pues, fundamental.

De igual manera, la rehabilitación deportiva se fundamenta en la ciencia básica de la rehabilitación, puesto que la respuesta del cuerpo humano a un traumatismo sigue una secuencia temporal predecible y relativa a la severidad, extensión y tipo de afectación del tejido. Aunque un manejo adecuado tras la lesión y una intervención ortopédica y rehabilitadora a tiempo, en adición a la buena salud del atleta y a su adecuado estado psicológico y nutricional, pueden optimizar las condiciones para la cicatrización y recuperación, en realidad no existe un factor absoluto que acelere este fenómeno.

La respuesta inicial a un traumatismo es la inflamación. Sin la respuesta inflamatoria, la normal cicatrización no puede realizarse, 
dado que la inflamación dispara los factores locales y sistémicos que inician el proceso de reparación y sustitución del tejido afectado, con la consecuente restauración de la función. La lesión primaria resultante de la acción traumática afecta a la integridad del tejido, produciendo lo que usualmente se refiere como lesión secundaria o hipoxia. Se inicia mediante la liberación de mediadores químicos por parte de las células afectadas, los cuales alteran el tono vascular y la permeabilidad capilar, permitiendo al plasma sanguíneo con sus proteínas el escapar hacia el tejido circundante para producir el característico edema. La siguiente respuesta corporal es la concentración de elementos defensivos, que usualmente ser encuentran en la circulación, en el sitio de la lesión. Una vez que los macrófagos completan la fagocitosis de los detritus procedentes del proceso inflamatorio, el proceso de reparación puede comenzar. Es importante reseñar que la inflamación es necesaria para la reparación, pero la verdadera regeneración del tejido no puede realizarse hasta que la primera decrezca. Si no se proporcionan las condiciones para que esto se produzca, bien por la aplicación de carga antes de tiempo o bien por la utilización de medios terapéuticos no adecuados, se puede dar una condición crónica de inflamación a menudo resistente al tratamiento. Por otra parte, una duración excesiva del periodo de inmovilización da lugar a una atrofia muscular y a una inhibición neural refleja que condicionan un progresivo daño articular, lo que muestra la absoluta necesidad de moderar los síntomas causados por la inflamación y de mantener el periodo de inmovilización dentro de un mínimo ${ }^{2}$. De hecho, la gestión de la intensidad y de los momentos de introducción de cargas de trabajo constituye un aspecto primordial y delicado en rehabilitación deportiva, dado que la urgencia en los tiempos de recuperación requeridos descarta la utilización de las pautas conservadoras propias de la rehabilitación para la población sedentaria.

En el proceso de reparación, los tejidos no viables son reemplazados a través de un proceso de proliferación fibroblástica, estableciéndose un puente con una densa red de capilares y de tejido conectivo. El colágeno se deposita en esta matriz, formando el tejido de cicatrización. Además de este tejido de reparación, se regenera parte del tejido original, de modo que en el resultado final ambos tipos de tejido coexisten. Un tratamiento apropiado permite un mayor porcentaje de tejido regenerado, y por tanto funcional, sobre el tejido fibroso de cicatrización.

El fin de la fase de proliferación fibroblástica marca el inicio de la llamada fase de maduración. En esta fase, el original patrón desorganizado de disposición de las fibras de colágeno cambia a un alineamiento.conforme a las líneas impuestas por el estrés físico, 
resultando en una organización de superior fuerza tensil. La restauración final de la integridad del tejido hasta el estado anterior a la lesión es altamente variable en tiempo, y es todavía una de las principales áreas de investigación en medicina deportiva. No cabe duda de que el avance en el conocimiento de la historia natural de la lesión es decisivo para el desarrollo de estrategias de tratamiento rehabilitador eficaces y rápidas, las cuales deberían estar en todo momento basadas en los hechos científicamente probados, más que en experiencias individuales aisladas.

Las lesiones deportivas frecuentemente requieren descanso, en orden a respetar los tiempos de reparación. Sin embargo, el deportista normalmente mantiene o mejora la funcionalidad del sistema musculoesquelético a base de someterlo a estrés repetidamente a través del entrenamiento. De igual modo, la falta de entrenamiento o desuso causa rápidamente cambios nocivos en este sistema, con una rápida reducción de sus cualidades mecánicas. En contraste con la rapidez de la pérdida, la recuperación es más prolongada en el tiempo. La atrofia es el decremento en el tamaño del tejido, y la medida de la circunferencia de la extremidad es una medida comúnmente usada que proporciona cierta idea de la extensión de la atrofia muscular. Sin embargo, los efectos de la inmovilización son numerosos y afectan directamente al rendimiento, ya que no se limitan al sistema musculoesquelético, sino que también afectan al sistema cardiovascular. Por consiguiente, es un continuo reto para el equipo de rehabilitación el minimizar los efectos de la inmovilización para agilizar el retorno seguro a la actividad.

La ultraestructura del músculo inmovilizado desarrolla alteraciones casi inmediatamente. Conforme el tiempo de desuso avanza, se producen cambios como un descenso en la síntesis de proteínas, en la capacidad oxidativa, en las propiedades contráctiles y en el área transversal, con un aumento relativo del colágeno intramuscular, lo que incide en un déficit de fuerza muscular. En los ligamentos, se observa un descenso de las propiedades mecánicas y funcionales con déficit de fuerza tensil y de elasticidad, debido a la reducción de la masa y densidad del colágeno, y a un aumento de su desorganización. En el hueso, se desarrolla osteoporosis y alteraciones en la unión hueso-ligamento, mientras que en las articulaciones se observa una reducción en la síntesis de proteoglicanos por parte del cartílago, con una disminución en el contenido en agua y una proliferación de tejido lipofibrótico dentro del espacio articular, lo que da lugar a una creciente rigidez. Estos cambios pueden ser reducidos por medio de técnicas tales como el 
estiramiento muscular, la estimulación transcutánea o la movilización pasiva continua.

El trabajo muscular es una piedra angular en rehabilitación deportiva, y la fisiología de la contracción muscular debe tenerse presente en los protocolos de recuperación. La idea de que los humanos pueden activar todas las unidades motoras de un músculo en una contracción máxima ha sido propuesta, aunque éste no suele ser el caso. El reclutamiento motor es menor en las contracciones excéntricas máximas que en las concéntricas. Paradójicamente, es posible ejercer una fuerza mayor en la contracción excéntrica. De igual modo hay diferencias entre las contracciones máximas voluntarias de músculos agonistas y antagonistas, mientras que, por ejemplo, parece que el entrenamiento continuado e intensivo de la carrera causa una inhibición nerviosa central de la contracción máxima voluntaria de los extensores de rodilla. De este modo, varios factores afectan a la calidad de la contracción muscular, si bien se acepta que la producción de máxima fuerza se relaciona directamente con el área muscular transversal. Aparte de este hecho, las variaciones en el reclutamiento de unidades motoras afectan significativamente a la producción máxima de fuerza. De becho se cree que la respuesta inicial al entrenamiento de fuerza e la adaptación neural, más que el cambio hipertrófico del músculo en si. Histe fenómeno podría explicar las ganancias de fuerza bilaterales que se producen tras el entrenamiento unilateral de una extremidad. De igual modo, este reclutamiento puede comprometerse en condiciones patológicas. Por ejemplo, la asociación clínica entre el hidrartros de rodilla y la atrofia de cuádriceps puede ser explicada en parte por la inhibición neural que motiva esta efusión sobre las motoneuronas del cuádriceps.

El músculo esquelético es un tejido extremadamente adaptable. $\mathrm{Al}$ igual que el desuso motiva la atrofia muscular, el incremento de carga por medio del entrenamiento de fuerza resulta en un incremento del tamaño muscular conocido como hipertrofia. Esta hipertrofia es dependiente de la intensidad, duración y frecuencia del entrenamiento, así como de la composición fibrilar y del estado de entrenamiento del sujeto. La rehabilitación muscular incluye el desarrollo de hipertrofia, pero también de otras cualidades musculares. La resistencia muscular ha sido descrita como lo opuesto a la fatiga, y esta fatiga muscular es el resultado de varios mecanismos fisiológicos. Se caracteriza por un agudo descenso del rendimiento motor manifestado por un incremento de la sensación de esfuerzo necesario para mantener un determinado nivel de fuerza o bien por un decremento involuntario del nivel de fuerza ejercido. Paradójicamente, la atrofia muscular, aún 
teniendo un gran efecto sobre la fuerza contráctil absoluta, no tiene una acción tan drástica sobre la fatigabilidad muscular, lo que debiera tenerse en cuenta en los programas de tratamiento.

Otro aspecto clásico y fundamental del trabajo de rehabilitación es la mejora del arco de recorrido de una articulación («Range of Motion» - ROM) en todos los planos. En condiciones no patológicas, el ROM puede estar limitado por la extensibilidad del tejido muscular y tendinoso, por las restricciones ligamentosas, y por la rigidez capsular. Después de una lesión, el ROM puede estar limitado por la efusión articular, el espasmo y contracturas musculares, y la acumulación de tejido de cicatrización en la articulación. Además, el ROM de un deportista puede ser muy diferente al de una persona sedentaria; baste pensar en la cadera de las gimnastas o en el hombro del lanzador.

Las limitaciones en el ROM están en función de las características de viscoelasticidad del tejido conectivo que forma parte de músculos, tendones y ligamentos. Este tipo de respuesta a la carga se caracteriza por una deformación elástica instantánea y proporcional a la carga, y por una deformación viscosa proporcional a la velocidad con que esta carga se aplica. El estiramiento produce cambios en la extensibilidad del tejido conectivo, el cual responde mediante sus características de «creep», o elongación en respuesta a una fuerza tensil fija, y relajación de estrés, o disminución de la tensión del tejido con el tiempo en respuesta a esta fuerza tensil fija. El reconocimiento de estos fenómenos forma la base para las técnicas de estiramiento y de ganancia de ROM.

\section{Diagnóstico clínico y funcional en rehabilitación deportiva}

La evaluación en rehabilitación deportiva comparte con la de la rehabilitación convencional el carácter funcional, que proporciona la base para el manejo del paciente a partir del diagnóstico clínico. Este diagnóstico clínico puede estar ya realizado cuando el deportista es remitido al equipo de rehabilitación, de manera que éste realiza la evaluación complementaria precisa para el diseño de los objetivos funcionales y plan de tratamiento. En el caso del deporte, este tipo de evaluación suele ser compleja, como lo son los requerimientos de la actividad del paciente y las prestaciones que debe efectuar el aparato musculoesquelético del deportista.

Cualquier tipo de prueba en medicina debería cumplir unas premisas fundamentales de fiabilidad y validez. En el caso del deporte, la validez 


\section{La rehabilitación en el deporte}

de una prueba es correlacionada frecuentemente con el rendimiento del deportista en pruebas atléticas estandarizadas. Asimismo, en el ámbito deportivo, los requerimientos de sensibilidad y especificidad para los tests de evaluación suelen ser mayores que en la rehabilitación convencional.

El diagnóstico funcional básico en rehabilitación puede comenzar con la evaluación de los parámetros básicos de fuerza muscular, arco de movimiento y propiocepción. Su medida sirve para valorar las repercusiones funcionales causadas por una patología y para monitorizar la progresión del deportista lesionado en el tiempo. También actúa como herramienta motivacional para el deportista, en cuanto comprueba el cumplimiento progresivo de las metas parciales fijadas por el equipo de rehabilitación.

La evaluación de la fuerza muscular se ha usado para estimar la integridad y función del sistema musculoesquelético desde finales del siglo pasado. Los instrumentos usados clínicamente se pueden resumir en: la evaluación manual, la cual es subjetiva y adolece de falta de fiabilidad y sensibilidad; los dinamómetros de mano, los cuales son mecánicamente fiables, gozando también de buena fiabilidad intra-observador e inter-observador en manos de evaluadores experimentados; los dinamómetros isocinéticos, que permiten una evaluación dinámica y cuyos resultados son fiables si se siguen protocolos estandarizados; y los dinamómetros isométricos, cuyas medidas son fiables pero estáticas, y se correlacionan altamente con la producción de fuerza isocinética a bajas velocidades. Todos estos sistemas requieren la aplicación del máximo esfuerzo por parte del sujeto evaluado si se quieren obtener registros máximos y reproducibles. A estos sistemas clínicos se pueden añadir otros propios del medio deportivo, en forma de diferentes tests dinámicos con pesos libres, aparatos de resistencia fija o variable, o ejercicios de autocarga, adaptados a partir de la evaluación de fuerza muscular en el deporte. De igual manera, los parámetros a evaluar varían con respecto a las mediciones habitualmente usadas en rehabilitación convencional. Debido a los requerimientos complejos de fuerza muscular del deportista, es importante la utilización de medios para medir diversas áreas de esta cualidad, en relación siempre con los objetivos finales y de acuerdo a la especialidad deportiva en cuestión, de modo que se establezcan las bases para el trabajo específico y restitución de éstas áreas. No se debiera de perder de vista que las características de fuerza muscular tienen una importante relación con la función y el rendimiento. Sin embargo, se ha prestado relativa poca atención a la validez de las mediciones de fuerza en este contexto, 
aunque sí es cierto que diferentes autores han relacionado positivamente el registro de valores de fuerza con diversas acciones funcionales deportivas.

En rehabilitación deportiva, el concepto clínico tradicional de medición del arco articular se debe completar con la medición precisa de la flexibilidad de una articulación. Podríamos englobar estos dos conceptos en la evaluación de la movilidad de una articulación, que incluye también la medición de las traslaciones articulares cuando tienen significación patológica (artrometría de la traslación sagital de la rodilla, por ejemplo). Esta movilidad articular debiera situarse dentro de unos límites de normalidad, diferentes para cada deporte, fuera de los cuales podríamos hablar de patología susceptible de actuación. Por ejemplo, unos isquiotibiales cortos pueden ser un factor de riesgo para el desarrollo de pubalgia en algunos deportes, al igual que una laxitud articular generalizada se relaciona con un mayor índice de lesiones de ligamentos en otros. De este modo, existe una relación directa demostrable entre alteraciones de la movilidad articular en alguno de sus componentes y la aparición de lesiones en el deportista. En la evaluación rehabilitadora del deportista, es exigible el registro de pruebas de flexibilidad de, al menos, las articulaciones y segmentos más relacionados con la patología. Una batería de tests en este sentido es de ayuda.

La propiocepción es el sentido de la posición articular y del movimiento articular (kinestesia). Es mediada por receptores en la piel, músculos y articulaciones, y juega un importante papel en el rendimiento deportivo. Además, está comprobado que mejoras en la propiocepción reducen el riesgo de lesión. De este modo, la medida de la capacidad del deportista para detectar cambios en la posición de sus articulaciones y reaccionar ante ellos corrigiendo su postura es un aspecto importante en rehabilitación deportiva, lo que ha llevado al desarrollo de diferentes tests, la mayoría de los cuales basados esencialmente en la estabilometría. El problema de estos tests suele ser que miden la sensación propioceptiva conjuntamente con aferencias de sensibilidad superficial cutánea, así como información procedente de los órganos visuales y laberínticos, de modo que lo que se toma a veces como valoración propioceptiva es en realidad valoración del equilibrio, la cual puede estar alterada como resultado de la afectación de alguna de estas aferencias. Así pues, para medir el estado propioceptivo de un deportista, alterado por una lesión, mediante un test de equilibrio, se debe de asumir la integridad de los otros sistemas neurológicos que intervienen en este sentido. Algunos sistemas modernos de valo- 
ración del equilibrio, modifican las vías vestibular y ocular para medir de manera más precisa el mecanismo propioceptivo, pero su verdadero valor está todavía por demostrar, dado su elevado costo y la ausencia de valores normativos. Mientras tanto, algunos tests clínicos de equilibrio sencillos de realizar, permiten tener una idea del estado del control neuromuscular propioceptivo de una extremidad y de su evolución con el tratamiento.

La mayoría de los movimientos deportivos comprenden la acción integrada de segmentos corporales y la transmisión y absorción de fuerzas. Por ejemplo, el tronco forma una base de apoyo desde la cual se generan las fuerzas para chutar un balón de fútbol. Estas relaciones forman el concepto de cadena musculoesquelética. Este concepto se demuestra bien en condiciones patológicas, en las cuales un defecto en una eslabón de la cadena puede afectar "a distancia» a otros eslabones. La asociación entre lesión distal y afectación proximal es un hallazgo común en lesiones de extremidad inferior. La evaluación clínica o instrumentada de las alteraciones de la biomecánica de los segmentos musculoesqueléticos, en reposo o bien durante la actividad deportiva en condiciones de laboratorio o de campo, debiera ser de rutina en la valoración diagnóstica en rehabilitación deportiva. Esta evaluación se puede focalizar en diferentes áreas de estudio, como el análisis de la marcha, de la pisada o de la alineación de los segmentos corporales, para lo cual se dispone de equipamiento más o menos sofisticado. De igual manera, es necesario el conocimiento adecuado de los gestos deportivos de cada especialidad, así como de las cargas que se ejercen y de las demandas funcionales de cada disciplina deportiva. La demanda funcional de un tobillo de un saltador de triple no tiene nada que ver con la de un nadador, por poner un ejemplo.

Incluso en presencia de buenos registros en la valoración clínica básica de fuerza muscular, arco de movimiento y propiocepción, y una vez corregidos los déficits biomecánicos, un deportista lesionado puede tener todavía importantes lagunas en el rendimiento funcional del segmento musculoesquelético que está rehabilitando. Los llamados tests funcionales evalúan conjuntamente el control neuromuscular, la fuerza, la potencia y la habilidad funcional. Deben de tener la misma exigencia en cuanto a estandarización, validez, fiabilidad, sensibilidad y especificidad, que cualquier otro test clínico, y usar datos de poblaciones muy definidas en orden a proporcionar comparaciones válidas en el ámbito deportivo.

Diversos autores han desarrollado tests funcionales. Los más utilizados para la extremidad inferior son los tests de salto, como el 
salto vertical y el de longitud. También se utilizan tests de carrera y cambio de dirección. Por otro lado, existen tests estandarizados de condición física que, debido a que requieren una integridad funcional del aparato locomotor, pueden utilizarse para evaluar el nivel alcanzado en el proceso de rehabilitación del deportista. Entre ellos se cuentan tests de rendimiento anaeróbico como el tests de Wingate o el de Margaria-Kalamen, o tests de agilidad, como el de figura de ocho o el de zigzag de Barrow. Estos tests funcionales no sólo miden fuerza muscular, sino también otros factores que contribuyen al rendimiento físico, como el dolor, edema, integridad ligamentosa, flexibilidad, coordinación, agilidad, potencia y propiocepción. Cualquiera de estos tests debe demostrar su capacidad de discriminación entre grupos patológicos y sanos. A este respecto y como ejemplo, es interesante destacar que algunos autores (Lephart et al, 1992) han encontrado que las medidas clínicas convencionales, incluyendo la evaluación de fuerza y la artrometría de traslación sagital, se correlacionan poco con la capacidad de un sujeto afecto de una deficiencia en el ligamento cruzado de la rodilla, para volver a los niveles deportivos prelesionales. Sin embargo, los deportistas que retornaban a estos niveles puntuaban significativamente mejor en los tests funcionales que estos autores utilizan, comparados con los que no podían retornar a su nivel deportivo previo.

Entre los problemas en la aplicación de los tests funcionales se encuentran la escasez de estudios bien diseñados acerca de su fiabilidad y validez. Además, existe poca estandarización, lo que hace la comparación de resultados es a veces difícil o imposible. Estos tests deben ser seleccionados en base a su facilidad de aplicación y equipamiento necesario, e incorporación del mayor número de destrezas relacionadas con el rendimiento en una especialidad deportiva. Por otro lado, no se conocen tests válidos y fiables para la extremidad superior o el tronco. En rehabilitación deportiva, es necesario determinar la capacidad de regreso a la competición de forma segura y eficiente. Esto no es posible si se utilizan tests no funcionales o no probados. Por tanto, este es un área de desarrollo fundamental en la disciplina que nos ocupa.

Durante el desarrollo del proceso de rehabilitación, es necesaria la planificación de los controles médico-clínicos pertinentes, y el uso de cuantas pruebas complementarias sea preciso. El diagnóstico por la imagen en particular ha conocido un importante desarrollo en los últimos tiempos, y los avances en ecografía musculoesquelética permiten controles seriados poco costosos de la evolución de muchas patologías. El uso de estas y otras pruebas complementarias, así como la plani- 


\section{La rehabilitación en el deporte}

ficación de controles clínicos y la coordinación de las actuaciones de otros especialistas, como biomecánicos, psicólogos u ortopedas, permiten la monitorización y modificación del proceso de rehabilitación, que se convierte así, idealmente, en un sistema cerrado basado en la evidencia.

Por último, y dado que el proceso de rehabilitación en deportistas cuida los aspectos de condición física, en aras de una reintegración a la actividad al mejor nivel deportivo posible, se hace necesaria la evaluación seriada de estas capacidades físicas, y la actuación en consecuencia. La pérdida de capacidad aeróbica y la ganancia de porcentaje adiposo es un hallazgo demasiado común en deportistas que terminan su proceso de rehabilitación de una lesión. Una evaluación física completa antes del alta definitiva nos indicará si el trabajo integral de rehabilitación deportiva ha sido efectivo.

\section{Terapéutica en rehabilitación deportiva}

La evaluación diagnóstica y funcional del deportista lesionado permite la definición de unos objetivos rehabilitadores - «cosas que hay que arreglar»- precisos hacia cuya consecución deben dirigirse las medidas terapéuticas que se elijan. La terapia en rehabilitación deportiva es de características complejas, y requiere el concurso de uno o varios profesionales dedicados en contacto diario con el deportista. Podría decirse que las técnicas de fisioterapia son la piedra angular del tratamiento, y en este aspecto es necesario destacar de nuevo las profundas diferencias entre la fisioterapia digamos «convencional» y la fisioterapia deportiva. La fisioterapia deportiva debiera alcanzar el mayor grado de especialización, parecida a la fisioterapia de la lesión traumatológica del sedentario tanto como se parece un taller mecánico de ciudad a un «box» de fórmula uno. El diseño y aplicación de la fisioterapia en el deporte es un asunto complejo, dado que la elevada demanda funcional del deportista requiere personal entrenado al respecto. Un gran número de opciones terapéuticas está disponible, y su número aumenta día a día. El manejo acertado de los medios térmicos, la electroterapia, la hidroterapia y la terapia manual, entre otras modalidades, proporciona los mejores resultados cuando su uso se orienta por medio de unos claros objetivos de tratamiento definidos en el plan inicial y basados en una cuidadosa evaluación del deportista. La constante aparición y desarrollo de alternativas de terapia, demanda un conocimiento, evaluación y aplicación de éstas por parte del terapeuta, proceso que, sin duda, debería basarse en criterios de evidencia científica y médica. 
La fisioterapia se complementa con otras aportaciones terapéuticas que se encaminan al tratamiento integral del deportista. La adecuada medicación local o general, la provisión de ortesis y equipamiento protector, y la programación del entrenamiento y rehabilitación funcional del deportista durante el proceso rehabilitador por medio de la coordinación de los entrenadores y personal especializado pertinente, así como la integración de otras posibles actuaciones de diversos tipos de profesionales que atiendan a consideraciones especiales del deportista en base a su edad, sexo o problemas patológicos intercurrentes, precisan una coordinación médica general, así como una evaluación continua. La responsabilidad sobre la evolución médica del deportista lesionado, tanto ante su entrenador y su entorno como ante el deportista mismo, debiera ser del médico coordinador de todo el proceso, tal como ocurre en cualquier proceso patológico en medicina.

En el medio deportivo, el objetivo final del programa de rehabilitación es la reincorporación rápida y segura a la actividad. Aunque es virtualmente imposible el acelerar el normal proceso de cicatrización que sigue a una lesión, se puede hacer mucho para optimizar el entorno en el que esta cicatrización ocurre, asegurando que nada impide este proceso. Para lograr este objetivo, el deportista debería participar en la fase de la rehabilitación llamada comúnmente Rehabilitación Funcional. El programa de rehabilitación funcional toma en cuenta las fases normales de reparación tisular, mientras que prepara al deportista, a través del trabajo de sus necesidades específicas, para las demandas que encontrará al reintegrarse a la competición. De este modo, este concepto se integra con el resto de los elementos que componen la estructura de rehabilitación deportiva ${ }^{3}$.

El equipo de rehabilitación deportiva, multidisciplinar en todo lo posible, identifica los objetivos clínicos y funcionales para el deportista. Los primeros se caracterizan por ser medibles en condiciones clínicas, y se refieren a parámetros como fuerza, arco de movimiento, inflamación, dolor, resistencia cardiovascular o resistencia muscular. Los objetivos funcionales se centran en los factores requeridos por el deportista para rendir en su deporte, y se refieren a parámetros como la velocidad, potencia, agilidad, control neuromuscular del movimiento, estabilidad neuromuscular de las articulaciones, y resistencia deporte-específica. Estos parámetros se miden por medio de tests funcionales.

La pregunta formulada con más frecuencia por un deportista lesionado es: ¿cuándo podré volver a entrenar normalmente? Dada la individualidad de cada deportista y lesión, cuando la respuesta es dada en términos de tiempo, por ejemplo, un número dado de días o semanas, 
el deportista podría retornar demasiado pronto o demasiado tarde. Sin embargo, estableciendo una serie de objetivos que el deportista debe cumplir antes de progresar a la siguiente fase del programa, el equipo de rehabilitación puede estar seguro de la seguridad de la vuelta a la actividad normal. Esta aproximación permite igualmente el entendimiento por parte del deportista del papel que él mismo juega dentro del programa, lo que aumenta su implicación. La obtención de éxitos parciales dentro del programa estimula la motivación del paciente y reduce su sentimiento de desánimo por no participar en su deporte, a la vez que evita la «trampa temporal» en la que se puede caer («usted dijo que volvería a competir en dos semanas»). Por último, este tipo de enfoque mejora la información y la coordinación entre el equipo de rehabilitación, el entrenador y el entorno del deportista.

Para cumplir los objetivos seleccionados, se debe diseñar el programa en una progresión secuencial etapa tras etapa, con cada etapa exigiendo un mayor nivel de rendimiento. El tipo y severidad de cada lesión indica dónde debe comenzar esta progresión, pero el último paso es, en cualquier caso, el retorno a la actividad deportiva. Esto requiere una comprensión de las demandas impuestas al deportista por su prueba o especialidad. Lo que puede considerarse fuerza o arco de movimiento «normales» para la mayoría de los pacientes en rehabilitación probablemente se consideraría inadecuado para la mayoría de los deportistas. Es pues conveniente poner al deportista en un entorno de rehabilitación que simule los requerimientos de su deporte, lo que mejora directamente la calidad de su recuperación y reduce las posibilidades de recidiva de lesión.

Desde un buen principio del proceso lesional, en el que es necesario la descarga de la zona afectada, es posible el mantenimiento de la fuerza, flexibilidad del resto de los segmentos corporales y de la condición cardiovascular por medio del ejercicio alternativo no interferente con la cicatrización de la lesión, o bien mediante la modificación de la actividad deportiva en volumen, intensidad $u$ otros parámetros del ejercicio.

Existen numerosas actividades que se pueden usar como formas alternativas de ejercicio. El empleo de ejercicios de control de las piernas como las minisentadillas o las extensiones de tobillo puede iniciarse en las primeras fases, con progresión a ejercicios más complejos, y siempre funcionales. Igualmente el uso del ciclismo, la retrorehabilitación (ejecución de ejercicios en el sentido contrario al normal), el ejercicio de bajo impacto, el ejercicio en agua, y toda una serie de actividades que mejoran la propiocepción (sensibilidad de posición de 
una articulación y de su movimiento) por medio de diferentes dispositivos, son recursos que se pueden emplear.

De este modo, en adición al entrenamiento y restitución de las cualidades físicas llamadas clínicas, que clásicamente se han atendido en la rehabilitación convencional, se integra el trabajo de la velocidad, agilidad, potencia y destrezas específicas por medio de una batería de ejercicios progresivos entre los que se enfatizan los llamados ejercicios de cadena cinética cerrada, que se caracterizan por ser realizados con el extremo distal de la extremidad fijado, produciéndose el movimiento en los segmentos proximales. Este tipo de ejercicios producen mejoras neuromusculares de la velocidad, equilibrio y coordinación, además de producir un aumento de la estabilidad articular por medio de la coordinación neural de la musculatura que rodea a dicha articulación. En algunos casos, estos ejercicios se suman a los convencionales, pero en otros los reemplazan totalmente debido a sus mayores beneficios. En adición, la realización de este tipo de ejercicios provee la base para el desarrollo de la progresión hacia actividades deporte-específicas como esprintar, lanzar, fintar o saltar, de forma más temprana. La realización bajo condiciones de carga progresiva de este tipo de actividades marca el contenido de las últimas fases del programa de rehabilitación funcional.

\section{Conclusión}

La rehabilitación adecuada de las lesiones deportivas requiere secuencialmente (1) el inmediato y preciso diagnóstico de la naturaleza y severidad de la lesión con el reconocimiento de los tejidos específicos afectados; (2) la inmediata iniciación de los tratamientos apropiados dirigidos a minimizar los efectos secundarios de la reacción inflamatoria; (3) una secuencia de rehabilitación ordenada, incluyendo la aplicación de medidas terapéuticas y de carga progresiva para mejorar la reparación de las estructuras tisulares dañadas; (4) la integración de actividades funcionales para mejorar la restauración de los patrones de movimiento coordinado propios del deporte y especialidad del individuo; y (5) la ejecución satisfåctoria y confiada de las actividades específicas de la especialidad deportiva, con el adecuado control corporal a niveles similares a los anteriores a la lesión.

Soy fisioterapeuta - tú eres un «athletic trainer» - ella es las dos cosas - él es un médico del deporte - tú eres un médico general formado en deporte en el extranjero - aquel es un médico rehabilitador. 
A veces los profesionales que trabajamos en el medio deportivo estamos más preocupados de cuántas credenciales, títulos o cursos podemos poner en nuestro currículum, tendiendo a olvidar cuál debiera ser nuestro principal objetivo: la prestación de una asistencia sanitaria competente y de calidad al deportista. La rehabilitación deportiva es un gran paraguas donde se refugian numerosas áreas de especialización profesional, las cuales están más o menos implicadas en, o bien mejorar el rendimiento del deportista, o bien en cuidar de su patología. Con una experiencia en el entorno deportivo más o menos profunda, fisiólogos del ejercicio, biomecánicos, nutricionistas, psicólogos del deporte, entrenadores, preparadores físicos, enfermeros, médicos, fisioterapeutas o «athletic trainers», podrían todos legítimamente reclamar el ser "especialistas» en rehabilitación deportiva. Ciertamente, cada una de estas áreas puede realizar una buena contribución hacia el atleta. Es esencial para todos estos profesionales, la realización de una aproximación en equipo, lo que saca el máximo partido a sus conocimientos, talento y experiencia, y redunda en el mejor rendimiento del deportista ${ }^{4}$.

La rehabilitación de lesiones de aparato locomotor en deportistas es sólo un área dentro del gran cuerpo de conocimiento de la traumatología, de la rehabilitación y de la medicina deportiva. Idealmente, es un proceso activo y participativo, en el que el deportista es motivado para conseguir sucesivas metas a través de un proceso continuo altamente estructurado y a la vez altamente individualizado; proceso que debiera estar fundado científicamente en la anatomía, biomecánica, fisiología básica y del deporte, teoría del entrenamiento, y en la terapéutica rehabilitadora. La aproximación multidisciplinar es sin duda lo más deseable, siempre y cuando se consiga una buena coordinación de todos los esfuerzos en bien del paciente.

La carencia de oferta de formación especializada integral en rehabilitación deportiva ha fomentado la aparición de numerosas aproximaciones desde diferentes campos. Mientras no se consiga la homogeneización de criterios, es posible que la mejor solución sea el aunar y coordinar estas aproximaciones dentro de los equipos. Y quizá esta palabra, «equipo», sea la clave para la optimización, de cara al deportista y a su lesión.

\section{Notas}

1 ZaChazewski, J.; Magee, D.; Quillen, W. (1996): Athletic Injuries and Rehabilitation. Saunders, Philadelphia. 


\section{Jesús Olmo Navas}

2 NichOlas, J.A.; HeRshmanN, E.B. (1995): The lower extremity and spine in sports medicine. Mosby, St. Louis.

3 LEPHART, S.; HENRY, T. (1993): Functional Rehabilitation for que upper and lower extremity. Sports Medicine 26: 579-591.

4 Rockwell, J.; Prentice, B.; Peyton, R. (1996): Physical therapist's and Athletic Trainer's Perspective, en Zachazewski, J.; MAGEe, D.; QuIllen, W. (1996): Athletic Injuries and Rehabilitation. Saunders, Philadelphia. pp. xi-xii. 